\title{
Influenza A virus H1N1 associated pneumonia - acute and late aspects evaluated with high resolution tomography in hospitalized patients
}

\author{
Cristina Asvolinsque Pantaleão Fontes, ${ }^{1}$ Alair Augusto Sarmet Moreira Damas dos Santos, ${ }^{1,2}$ \\ Solange Artimos de Oliveira, ${ }^{3}$ Miquel Abdon Aidê ${ }^{3}$
}

${ }^{1}$ Radiology Service, Department of Radiology, Faculty of Medicine, Antônio Pedro University Hospital, Federal Fluminense University, Niterói

${ }^{2}$ Imaging Center of the Complex Hospital of Niterói (CHN), Niterói

${ }^{3}$ Department of Clinical Medicine, Faculty of Medicine, Antônio Pedro University Hospital, Federal Fluminense University, Niterói, Brazil

Background: Influenza A (H1N1) virus often compromises the respiratory tract, leading to pneumonia, which is the principal cause of death in these patients. The purpose of this study was to review the acute and late phase pulmonary findings in influenza $\mathrm{A}(\mathrm{H} 1 \mathrm{~N} 1)$ associated pneumonia using high resolution computed tomography (HRCT), and to determine the importance of performing end expiration series.

Methods: Between July and August 2009, 140 patients presented with influenza A (H1N1) confirmed by real-timepolymerase chain reaction. Out of these, 27 patients underwent HRCT in the acute and late phases of pneumonia, allowing for a comparative study. Late phase exams were performed due to clinical worsening and up to 120 days later in patients with persistent complaints of dyspnea.

Results: Ground glass opacities, consolidations, and the combination of both were associated with the acute phase, whereas persistence or worsening of the lesions, lesion improvement, and air trapping in the end expiration series (as seen using HRCT, $n=6$ ) were observed in the late phase.

Conclusions: In the HRCT end expiration series, air trapping was found in the late phase of H1N1 associated pneumonia. Generally, these exams are not evaluated in research articles, and air trapping has not previously been studied using the end expiration series. Our study brings more scientific knowledge about aspects of pulmonary involvement by influenza A (H1N1), through evaluation with end expiration series, which makes the CT exam dynamic, translating the respiratory movement, and showing bronchial alteration.

Key words: Influenza A(H1N1); viral pneumonia; HRCT; HRCT end expiration; air trapping; small distal airways.

Correspondence: Cristina Asvolinsque Pantaleão Fontes, Radiology Service, Antônio Pedro University Hospital, Rua Mem de Sá 169/1101, 24220-261 Niterói, RJ, Brazil.

Tel. +55.21.27113919,+55.21.976315260 - Fax:+55.21.26299076. E-mail: cfontes@id.uff.br

Contributions: CAPF and SAO contributed in designing the study, CAPF and AASMDS collected the data, analyzed by CAPF and MAA. The final report and the article were written by CAPF, AASMDS, SAO and MAA and the article was read and approved by all the authors.

Conflict of interest: The authors declare no conflict of interest.

Funding: We have not received any grants or funding for this work.

Availability of data and materials: Data available by contacting the corresponding author.

Ethics approval and consent to participate: Our study has got the permission approved by our institution's Medical Ethics Research Committee (Institutional Ethics Research Committee of Federal Fluminense University, Faculty of Medicine, Antônio Pedro University Hospital, under CEP CMM /HUAP15811 and CAAE: 0165.0.0258-11). Our retrospective observational study was approved without the need for consent to participate.

Consent for publication: Not applicable. 


\section{Introduction}

The influenza A (H1N1) virus is a recombination of influenza A genetic material from human, swine, and avian species and often causes involvement of the pulmonary parenchyma. Recently, high resolution computed tomography (HRCT) along with other advancements have led to the development of volumetric exams, aiding in the diagnosis and characterization of pulmonary disease. This study aimed to review the acute and late phase pulmonary findings in H1N1 using HRCT, and to determine the importance of performing end expiration series to complement the analysis of pulmonary parenchyma, because it is associated with the dynamics of breathing [1].

\section{Methods}

In this retrospective, descriptive, observational study, 140 patients were confirmed to have Influenza A (H1N1) viral infection by nasal swab and real time polymerase chain reaction (RTPCR). Out of these patients, 44 were admitted to our hospital, and ultimately, a total of 27 patients were included in the present study, who were persistent with pulmonary complaints of dyspnea and underwent HRCT during their stay in the hospital and follow up occurred for the duration of dyspnea, for a total of 64 exams. The study was approved by the Institutional Ethics Research Committee of our institution and as retrospective observational study without patient informed consent. Patients underwent chest CT exams in a multidetector scanner with 64 channels, with high resolution and end expiration series. All exams were evaluated by two radiologists with experience in thoracic radiology, and the tomographic findings were described according to the chest radiology consensus, making use of the terms for description used for chest CT [2]. In this study, the findings were divided according to the acute and late (10 days to 4 months after first onset of respiratory symptoms) phases of the disease, in patients with persistent pulmonary complaints. In the acute phase, the period between the onset of respiratory symptoms and the first HRCT ranged from one to nine days. In the late phase 37 exams were performed. To better evaluate the pulmonary findings in HRCT, we divided the lungs according to the coronal and axial planes. In the coronal plane we divided each lung into thirds, evaluating each third separately, according to previous studies [3]. The upper third was above the carina, the middle third was between the carina and the inferior pulmonary vein, and the lower third was distal to the inferior pulmonary vein. In the axial plane we evaluated the topography of the central and peripheral lesions [2]. We performed descriptive analysis of the findings and the results were expressed as counts and percentages, given the case series, complex statistical methods were not used.

\section{Results}

Out of 27 patients in the present study, 12 required ventilatory assistance in the intensive care unit, ten required an oxygen mask, and two required mechanical ventilation. The period of hospitalization in patients without comorbidities ranged from one to 15 days, and in those with comorbidities from two days to four months. All patients remained with respiratory restriction in the acute phase. Further, all patients were medicated with antiplatelet and antiviral drugs, in addition to other symptomatic medications, according to the clinical follow up by intensivist physicians. In our study, 11 patients had comorbidities; four had one comorbidity, and seven had more than one comorbidity. Two patients had human immunodeficiency virus/acquired immunodeficiency syndrome (HIV/AIDS), two had systemic lupus erythematosus, and one had polymyositis; all patients were being treated appropriately for the comorbidities. The one patient with lupus erythematosus was morbidly obese and had secondary antiphospholipid antibody syndrome. Both lupus patients used corticosteroids and hydroxychloroquine, and the patient with polymyositis used corticosteroids. Patients with HIV/AIDS were taking regular antiretroviral therapy medication and had mild lung changes, as shown by HRCT. Specifically, these patients had only parenchymal bands in the lower third of the lungs and unilateral ground glass opacity. Out of these 11 patients, six were men and five were women, and all presented with fever, cough, and myalgia, four presented with dyspnea, one with respiratory discomfort, three with complaints of arthralgia, and one with coryza and headache. The other comorbidities presented were systemic arterial hypertension, chronic obstructive pulmonary disease, diabetes mellitus, cancer, and one patient was a smoker. In the patients with HIV/AIDS, we observed reduction of the lesions in the late phase in both patients, and one patient had air trapping. The most common findings observed with HRCT in the acute phase of the disease were consolidations predominantly in the lower and peripheral thirds of the lungs (12 patients, $32.4 \%$ ), ground glass opacities ( 5 patients, $13.5 \%$ ), and both consolidations and ground glass opacities (10 patients, 27\%) (Table 1). At the

Table 1. Findings regarding variables and frequency in different aspects related to interval between beginning of the symptoms and achievement of HRCT, hospitalization, mechanical ventilation, comorbidities, HRCT aspects on the acute and late phase, of the 27 patients.

Variable

Interval between beginning of the symptoms and achievement of HRCT

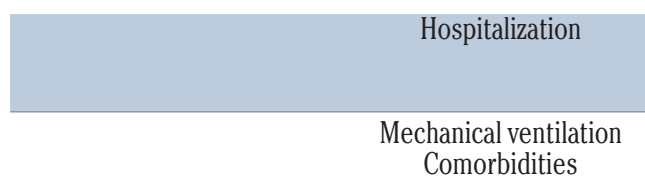

HRCT findings on the acute phase (1st exam)

HRCT findings on the late phase (2nd exam)
Frequency

1 to 4 days - 15 cases

5 to 9 days - 12 cases

1 to 10 days - 17 cases

11 to 20 days - 6 cases

above 21 days - 4 cases

2 cases

11 cases

(one comorbidity - 4 cases)

(more than one - 7 cases)

Consolidations - 12 cases

Ground glass opacities - 5 cases

Both findings - 10 cases

Lesions reduction - 24 cases

Persistence - 1 case

Worse - 1 case

Resolution - 1 case 
start of pandemic, the Emergency team routinely requested chest and sinus X-rays to assess the flu status in acute phase, but not all patients underwent chest X-rays because in the course of the pandemic, the Emergency team already had started with requests from chest CT exam. The findings of the CT exam already helped in the diagnosis, ruling out or corroborating clinical suspicion, and also in the need for isolation of the patient.

At the second exam (conducted in the late phase) 24 patients had reductions in lesion size, one had persistence, one had worse- ning, and one had resolution. In this phase, four patients who presented with consolidations at the initial exam showed ground glass opacity. Two cases $(7.4 \%)$ showed a worsening in pulmonary involvement with HRCT, and presented with ground glass opacities, more extensive consolidations, and one patient had pleural effusion. Thirteen cases (48.1\%) presented with improvement of the pulmonary lesions in the late phase during the second exam, specifically, the lesions showed less extension of the parenchymal involvement and had a lower density. We observed the presence of

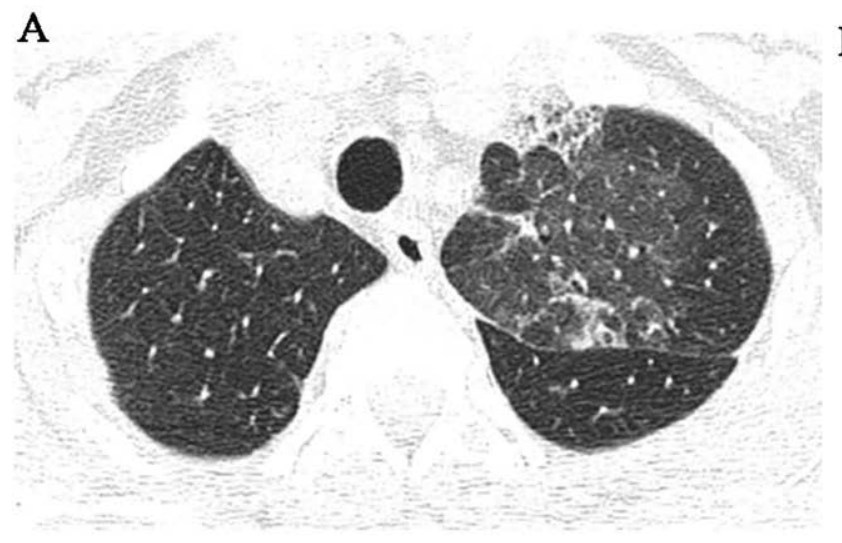

B

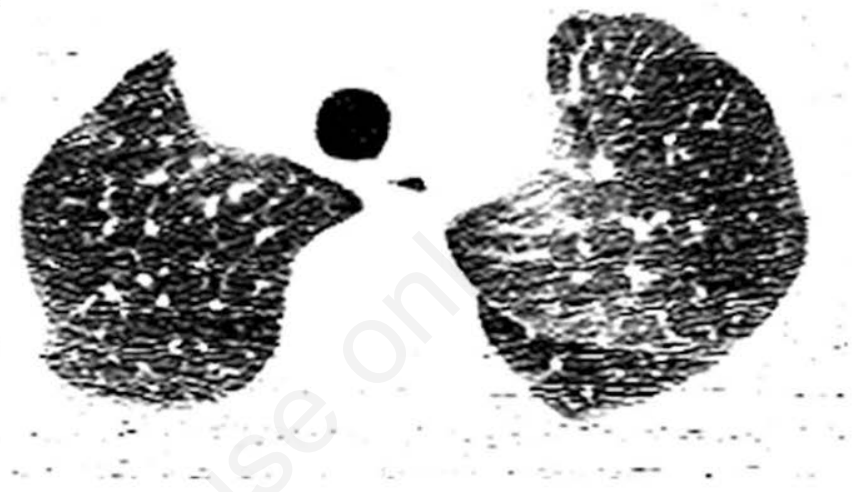

Figure 1. A) HRCT with areas of ground glass opacities in the superior third of left lung on 25 July 2009, in a patient who evolved with complaints of mild dyspnea. B) Follow up exam on 29 September 2009, with improvement of these findings, but with the appearing of areas of air trapping, better evidenced in end expiration HRCT (case 11).

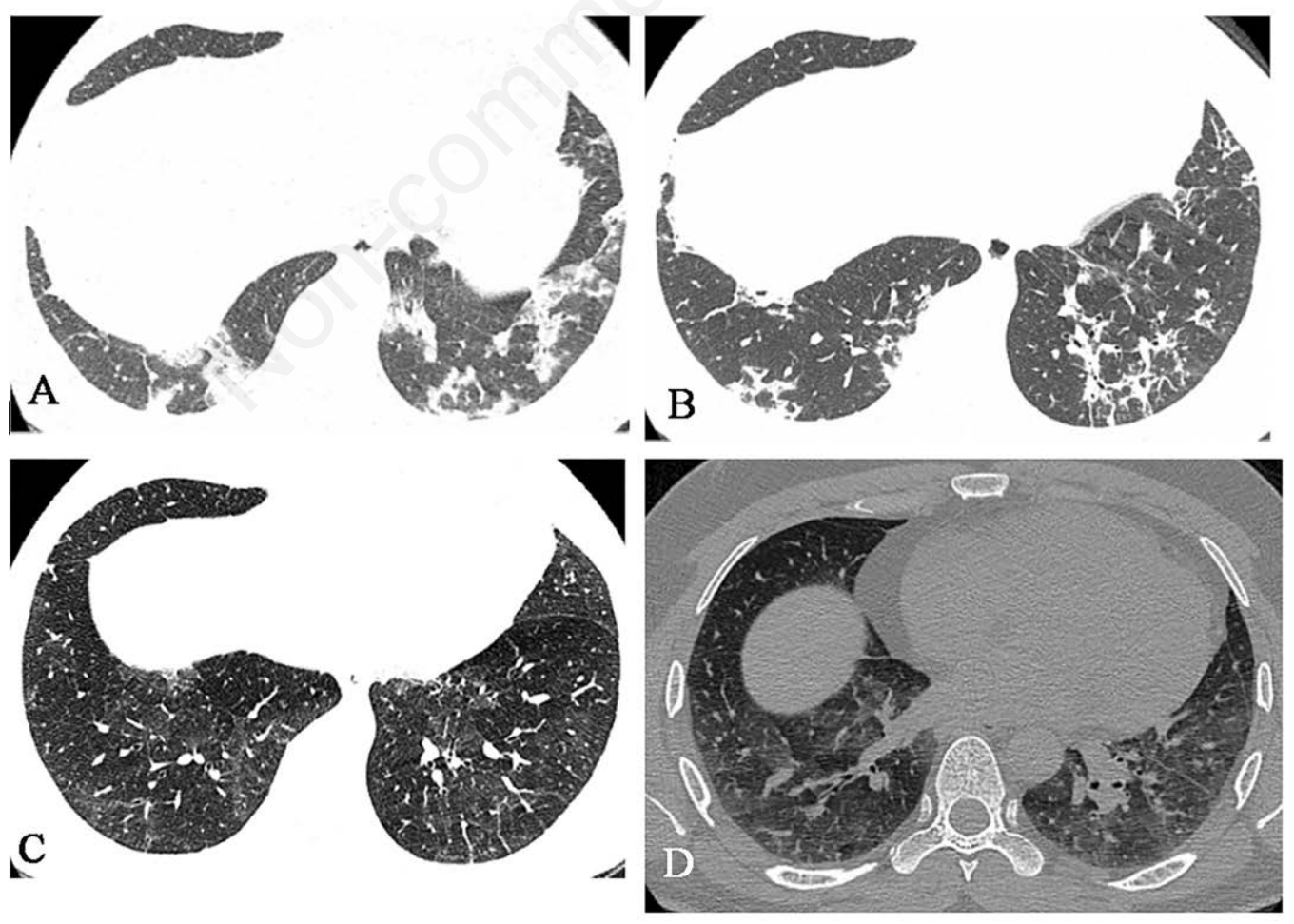

Figure 2. A) HRCT shows areas of consolidation and ground glass opacities in the lower third of both lungs, predominating in the periphery, in a patient who evolved with complaints of dyspnea (7 August 2009). B) Follow up exam (13 August 2009) discriminating the improvement of pulmonary findings, translated to smaller dimensions and density of the lesions, and in (C) (21 September 2009) with improvement of these findings, but with the appearing of areas of air trapping, better evidenced in end expiration HRTC in (D) (5 October 2009) (case 4). 
parenchymal bands in one patient in the acute phase, and in four patients in the late phase in the second and third exams.

Patients who presented with ground glass opacities, with or without consolidation, showed greater involvement of the lungs in the late phase in two cases, persistence of the lesions in 12 cases, and resolution of the lesions in 13 cases. Among those who presented complete resolution of pulmonary parenchymal lesions, 6 cases $(22 \%)$ showed areas with air trapping, which was more evident at end expiration (Figures 1 and 2).

These six patients had persistent complaints of mild dyspnea, and four began corticosteroid therapy. One patient had air trapping in the late phase at the second exam, four patients had air trapping at the third exam (range: 47 to 61 days, mean of 52.2 days), and one patient had air trapping at the fourth and fifth exams, with 76 and 96 days, respectively. In all patients, air trapping was observed in previously affected areas. Other findings, such as mosaic paving patterns, septal thickening, thickening of bronchial walls, perilobular interstitial thickening, and bilateral pleural effusion were seen less frequently.

\section{Discussion}

Tomographic findings were similar to those of seasonal influenza, other viral pneumonias associated with adenovirus, respiratory syncytial virus and parainfluenza, and severe acute respiratory syndrome (SARS) caused by coronavirus (SARS-Cov-1) infection in the year 2003 [4-10].

The histopathological lesions of this infection are similar to pneumonia caused by other influenza viruses [10,11], with diffuse pulmonary lesions affecting the epithelium and, in the most severe cases, resulting in bronchitis and/or bronchiolitis with necrosis and diffuse alveolar damage (DAD) $[4,6,12]$. In the late phase of the disease, the organization DAD, fibrosis, type II pneumocyte hyperplasia, epithelial regeneration, and squamous metaplasia are similar to the fibroproliferative phase of severe acute respiratory syndrome $[6,13,14]$, and findings on HRCT exams will depend on the pathological process that affects the lung.

Hyperinflation is common in viral pneumonias due to associated bronchiolitis, but in cases of influenza A (H1N1) infection, aerial entrapment appears to be a late consequence of DAD [10]. In one of the first published articles on the H1N1 pandemic, PerezPadilla et al. [11] reported that lung damage was likely due to the effect of the viral infection rather than a bacterial co-infection.

Regarding the topography of the lesions, in our study, HRCT showed bilateral involvement in $89 \%$ of patients. In the coronal plane, 15 patients had lesions that predominated in the lower thirds (40\%), and nine (24\%) had lesions that were diffuse. In the axial plane, nine patients $(24 \%)$ had lesions that were predominantly in the peripheral regions of the lungs. In the literature, lesions are typically reported to be bilateral, multifocal, and predominantly involve the lower lobes $[6,10,15-17]$. The data regarding parenchymal involvement vary in relation to the axial distribution, with reports of similar central or peripheral predominance $[16,17]$. Other less common findings in the present study include the mosaic paving pattern (1 patient), septal thickening not associated with ground glass opacities (four patients), and small bilateral volume pleural effusion (one patient), which has been described by other authors $[6,16,18]$. Six patients had bronchial wall thickening, which has also been described in some studies [7,16,19].

In one of the first article on this pandemic pneumonia, Lee et al. [20] described two patients: one with ill-defined ground glass opacity nodules and path areas of ground glass attenuation, thought to be typical pattern of viral pneumonia, and another one with secondary pneumococcal pneumonia which showed bilateral areas of lobar consolidation and ground glass opacities. These findings were somewhat different from typical pattern of viral pneumonia and were thought to have combined component of bacterial lobar pneumonia, demonstrating that HRCT can play an important role in the detection and characterization of this pneumonia.

We observed perilobular interstitial thickening in two patients in the late phase, as described by other authors [1], which has also been reported in organizing pneumonia $[21,22]$. Six patients $(22.2 \%)$ showed air trapping upon end expiration in the late phase, suggesting that the occurrence of air retention was due to distal airway obstruction. These findings were observed mainly in areas where there was major pulmonary involvement. Air trapping has been poorly reported in relation to $\mathrm{H} 1 \mathrm{~N} 1$ infection $[4,6,23]$, as most studies have not examined end expiration serie, hindering the study of respiratory dynamics. Acquiring the end expiration serie increases the exam time and requires patient collaboration, which is likely the reason why this sequence was not performed in most of the literature and does not appear in these retrospective studies. Despite the potential difficulties, this sequence must always be performed, as air trapping is an important aspect in patients with dyspnea complaints.

Li et al. [3] found that $13 \%$ of patients had a mosaic attenuation pattern and air trapping with a ventral distribution in the lungs, which was related to the use of mechanical ventilation. In our study, areas with air trapping were observed in regions previously affected by ground glass consolidations and opacities and were not related to the type of ventilatory therapy used. Out of six patients with air trapping who were followed up with in this study, all patients presented with dyspnea complaints, and four of them underwent corticotherapy with a good clinical response. Among the differential diagnoses suggested by the imaging findings, we observed similarities with the pulmonary manifestations of SARSCov-1 in 2003, described by Hui et al. [13] and Li et al. [3]. In hospitalized patients, abnormalities tend to progress to lesions with ground glass opacities and more extensive bilateral consolidations. These findings are similar to those observed in influenza A (H1N1), but without the predominance of the lungs' periphery. Regarding Sars-CoV-1, the impairment is more severe, and the appearance of the images may change daily [15].

In relation to recent publications on HRCT aspects of novel coronavirus SARS-Cov-2 (COVID-19) associated pneumonia the findings of ground glass opacities and consolidations predominating in the periphery have been described [24-26]. Pan et al. reported different aspects in sixty-three confirmed patients, such ground glass nodules, patchy/punctate ground glass opacities, patchy consolidation, fibrous stripes and irregular solid nodules [25]. The imaging aspects change rapidly in this novel viral pneumonia, and fifty-four patients in this serie progressed, including single ground glass opacities increased enlarged, and consolidated, fibrous stripe enlarged, while solid nodules increased and enlarged. In other study with 21 confirmed patients with COVID-19 Chung et al. found bilateral pulmonary parenchymal ground glass and consolidative pulmonary opacities, sometimes with a rounded morphology and a peripheral lung distribution [26]. Follow up exams were performed on eight patients and often demonstrated mild or moderate progression of disease, as manifested by increasing extent and density of lung opacities [26]. Therefore, there are also nonspecific findings for pulmonary involvement of this viral disease, although they have some similar findings of those seen in influenza A (H1N1) pneumonia, especially in the acute phase, but with notably peripheral injuries. Yu et al. compared findings in patients with mild and severe disease and they concluded that the most common findings were ground glass opacities with or without consolidation in a peripheral and basilar predominant distribution, and patients with severe form of the disease had more extensive opacification of the lung parenchyma than did patients with mild dis- 
ease, and noted that interlobular septal thickening, air bronchograms, and pleural effusions were also more prevalent in severe COVID-19 [27]. Comparing the most common findings in the acute phase of CT in H1N1-associated pneumonia, which are ground-glass opacities, consolidations and the combination of the two aspects, and in COVID-19 in which bilateral lesions predominate, with ground glass opacities, sometimes with rounded morphology, consolidations, reticular pattern, crazy paving and halo signs, we believe that the most important aspects in differentiating these diseases in the acute phase would be mainly the bilateral peripheral distribution and the rounded aspect of the lesions in COVID-19.

In relation to a comparative study carried out by Onigbinde et al. comparing the findings of COVID-19 and H1N1 influenza, the differences found in ground glass opacities report that in COVID19 they are more frequent in the periphery and in random topographies, and in influenza these lesions were more central [28]. Singh et al. conducted a prospective study in two patients who persisted with complaints of dyspnea following $\mathrm{H} 1 \mathrm{~N} 1$ pneumonia during the 2009 influenza pandemic. The patients were followed up with in 2015 and showed persistence of ground glass opacities and reticular opacities. The patients were diagnosed with residual pulmonary fibrosis, but expiration series was not performed [14]. Ishiguro et al. [19] conducted a study of 96 patients who had pneumonia associated with three types of influenza viruses [(A) H1N109pmd, $\mathrm{H} 3 \mathrm{~N} 2$, and B], and found that the aspect of primary viral pneumonia differed depending on the viral cause. They found that patients with influenza A pneumonia (H1N1pmd09) often had ground-glass opacities, which affected more lobes than patients with influenza A (H3N2) and pneumonia B. In addition, patients with H3N2 pneumonia had more frequently diffuse bronchial wall thickening. Neither the severity nor the mortality differed between the three subtypes of viral pneumonia [19].

In our study, two patients died. All other patients were followed up with and had a good clinical course. Regarding the improvement of the pulmonary lesions in the 25 patients that survived, four patients showed parenchymal bands in the late phase (two with previous consolidations and two with ground glass opacities). This latter finding has been described previously and should represent an aspect of organizing pneumonia [16,17], or secondary to the interstitial fibrosis process [12].

One of the limitations of our study was the comparative evaluation of the dates of the exams in the late phase since the exams were performed based on clinical worsening and at the request of the ICU medical team. Additionally, after hospital discharge the patients were followed up with by different pulmonologists. This study is important because several efforts are still being made to control the spread of influenza. Since the vaccine for H1N1 is not available everywhere, patients may experience delays in vaccination and/or refuse to be vaccinated [21].

In comparison with the acute and late stages of the disease. HRCT can help to predict the clinical prognosis to rule out other pulmonary complications or aspects of bacterial infections, aiding in the determination of the appropriate treatment.

\section{Conclusions}

The images obtained in our study in patients with influenza A (H1N1) pneumonia included areas of consolidation and/or ground glass opacities, unilateral or bilateral findings, and predominated at the periphery of the lungs. HRCT may reveal the involvement of the pulmonary parenchyma, predominantly in ground glass opacities. Patients who are cured may have complete resolution of lung parenchymal lesions or may experience air trapping in areas that are more evident in the end expiration series of HRCT. This suggests that air trapping can be found in the late phase after influenza (A) H1N1 infection, demonstrating the involvement of the small distal airways [22]. The imaging findings in the comparison with the acute and late phases of the disease may help to predict the clinical prognosis, and aid in the determination of the appropriate treatment strategy by eliminating other potential causes, such as pulmonary complications or bacterial infections.

Recent outbreaks of influenza A have been more frequent, and influenza $\mathrm{A}(\mathrm{H} 1 \mathrm{~N} 1)$ pdm09 virus responsible for the 2009 pandemic continues to be circulating. The increase in cases with lethal success shows the importance of early diagnosis and rapid institution of treatment, and the clinical-epidemiological correlation, laboratory and imaging tests are the most important actions in the acute phase of infection. As observed in the 2009 pandemic and subsequent outbreaks, pulmonary findings in X-ray and in CT have a direct correlation with the severity of the clinical picture, showing how much worse the pulmonary involvement, the worst is the clinical course, remembering that pneumonia is the leading cause of death in these patients $[13,23]$. Although now we have a molecular test with a viral panel that makes it possible to diagnose the etiology, besides viral or bacterial coinfection, (which was not available in 2009), cases with deaths are still frequent [29,30].

We believe that all laboratory and imaging methods should be used for rapid diagnosis and early institution of antiviral treatment, providing support in hospital care when necessary, reducing hospitalization time and avoiding the use of antibiotics.

\section{Acknowledgments}

We are grateful to the entire team involved in the care of patients with influenza A (H1N1) at the hospital and the diagnostic imaging service, that dealt with several patients almost above human capacity. As we feel unable to mention here all the participants, we would like to pay tribute to a colleague of ours who worked during the Influenza A (H1N1) pandemic and has now been victim of COVID-19, radiologist Victor Luís Bon.

\section{References}

1. Rubin GD. Computed tomography: revolutionizing the practice of medicine for 40 years. Radiology 2014:273:S45-74.

2. Hansell DM, Bankier AA, MacMahon H, McLoud TC, Müller NL, Remy J. Glossary of terms for thoracic imaging. Radiology 2008;246:697-722.

3. Li P, Zhang J-F, Xia X-D, Su D-J, Liu B-L, Zhao D-L, et al. Serial evaluation of high-resolution CT findings in patients with pneumonia in novel swine-origin influenza A (H1N1) virus infection. Br J Radiol 2010;85:729-35.

4. Tanaka N, Emoto T, Suda H, Kunihiro Y, Matsunaga N, Hasegawa $\mathrm{S}$, et al. 2012. High resolution computed tomography findings of influenza virus pneumonia: a comparative study between seasonal and novel (H1N1) influenza virus. Jpn J Radiol 30:154-61.

5. Verrastro CGY, Abreu Junior L, Hitomi DZ, Antonio EP, Neves RA, D'Ippolito G. Manifestations of infection by the novel influenza A (H1N1) virus at chest computed tomography. Radiol Bras 2009;42:343-8.

6. Abbo L, Quartin A, Morris M, Saigal G, Ariza-Heredia E, Mariane P, et al. Pulmonary imaging of pandemic influenza H1N1 infection: relationship between clinical presentation and disease burden on chest radiography and CT. Br J Radiol 2010;83:645-51. 
7. Ajlan AM, Quiney B, Nicolaou S, Müller NL. Swine-origin influenza A (H1N1) viral infection: Radiographic and CT findings. Am J Roentgenol 2009;193:1494-9.

8. Amorim VB, Rodrigues RS, Barreto MM, Zanetti G, Marchiori E. Computed tomography findings in patients with H1N1influenza A infection. Radiol Bras 2013;46:299-306.

9. Marchiori E, Zanetti G, Hochhegger B, Rodrigues RS, Fontes CA, Nobre LF, et al. High-resolution computed tomography findings from adult patients with Influenza A (H1N1) virusassociated pneumonia. Eur J Radiol 2010;74:93-8.

10. Son JS, Kim YH, Lee YK, Park SY, Choi CW, Park MJ, et al. Pandemic influenza (H1N1) viral pneumonia without co-infection in Korea: chest CT findings. Tuberc Respir Dis 2011;70:397-404.

11. Perez-Padilla R, de la Rosa-Zamboni D, Ponce de Leon S, et al. Pneumonia and respiratory failure from swine-origin Influenza A (H1N1) in Mexico. N Engl J Med 2009;361:6809.

12. Kim EA, Lee KS, Primack SL, Yoon HK, Byun HS, Kim TS, et al. Viral pneumonias in adults: radiologic and pathologic findings. Radiographics 2012;22:S137-49.

13. Hui DS, Joynt GM, Wong KT, Gomersall CD, Li TS, Antonio $\mathrm{G}$, et al. Impact of severe acute respiratory syndrome (SARS) on pulmonary function, functional capacity and quality of life in a cohort of survivors. Thorax 2005;60:401-9.

14. Singh N, Singh S, Sharma BB. Swine flu fibrosis: Regressive or progressive? Lung India 2016;33:219-21.

15. Hendrickson CM, Matthay MA. Viral pathogens and acute lung injury: investigations inspired by the SARS epidemic and the 2009 H1N1 influenza pandemic. Semin Respir Crit Care Med 2013;34:475-86.

16. Marchiori E, Hochhegger B, Zanetti G. Organizing pneumonia as a late abnormality in influenza A (H1N1) virus infection. $\mathrm{Br}$ J Radiol 2012;85:841-7.

17. Gómez-Gómez A, Martínez-Martínez R. Organizing pneumonia associated with swine-origin Influenza A H1N1 2009 viral infection. Am J Roentgenol 2011;196:W103-4.

18. Karadeli E, Koç Z, Ulusan S, Erbay G, Demiroğlu YZ, Şen N. Chest radiography and CT findings in patients with the 2009 pandemic (H1N1) influenza. Diagn Interv Radiol 2011;17:216-22.

19. Ishiguro T, Takayanagi N, Kanauchi T, Uozumi R, Kawate E, Takaku Y, et al. Clinical and radiographic comparison of influenza virus-associated pneumonia among three viral subtypes. Intern Med 2016;55:731-7.

20. Lee CW, Seo JB, Song J, Lee HJ, Lee JS, Kim MY, et al. Pulmonary complication of novel influenza A(H1N1) infection: imaging features in two patients. Korean J Radiol 2009; 10:531-4.

21. Quinn SC, Jamison AM, An J, Hancockc GR, Freimuthd VS. Measuring vaccine hesitancy, confidence, trust and flu vaccine uptake: Results of a national survey of white and African American adults. Vaccine 2019;37:1168-73.

22. Marchiori E, Zanetti G, Mano CM. Swine-origin influenza A (H1N1) viral infection: small airways disease. Am J Roentgenol 2010;195:W317; author reply W318.

23. Morens DM, Taubenberger JK. Making universal influenza vaccines: lessons from the 1918 pandemic. J Infect Dis 2019;219:S5-13.

24. Sverzellati N, Milone F, Balbi M. How imaging should properly be used in COVID-19 outbreak: an Italian experience. Diagn Interv Radiol 2020;26:204-6.

25. Pan Y, Guan H, Zhou S, Wang Y, Li Q, Zhu T, et al. Initial CT findings and temporal changes in patients with the novel coronavirus pneumonia (2019-nCoV): a study of 63 patients in Wuhan, China. Eur Radiol 2020;30:3306-9.

26. Chung, M, Bernheim A, Mei X, Zhang N, Huang M, Zeng X. CT Imaging features of 2019 novel coronavirus (2019-nCoV). Radiology 2020;295:202-7.

27. Yu M, Xu D, Lan L, Tu M, Liao R, Cai S, et al. Thin-section chest CT imaging of coronavirus disease 2019 pneumonia: comparison between patients with mild and severe disease. Radiol Cardiothorac Imaging 2020. doi: org/10.1148/ryct. 2020200126

28. Onigbinde SO, Ojo AS, Fleary L,Hage R. Chest computed tomography findings in COVID-19 and influenza: A narrative review. BioMed Res Int 2020;2020:6928368.

29. Centers for Disease Control and Prevention. 2018-2019 flu season: Flu activity elevated nationally. Available from: https://www.cdc.gov/flu/spotlights/flu-activity-elevated. htm\#: :text=December $\% 2021 \% 2 \mathrm{C} \% 202018 \% 20 \% \mathrm{E} 2 \% 80 \% 9$ $3 \% 20$ Increases $\% 20$ in, the $\% 202018 \% 2$ D2019\%20influenza $\% 2$ 0season

30. WHO. Global influenza strategy 2019-2030. Geneva: World Health Organization; 2019. Available from: https://apps. who.int/iris/handle/10665/311184 